\title{
Auditoria de contas em saúde: análise das glosas e suas justificativas no Home Care
}

\author{
Health Account Audit: analysis of disallowances and their justifications in the Home Care \\ Auditoría de cuentas de salud: análisis de desestimaciones y sus justificaciones en el \\ Home Care
}

Adam Carlos Cruz da Silva ${ }^{1 *}$, Vivian Schutz².

\section{RESUMO}

Objetivo: Analisar as glosas e as suas justificativas como o seu impacto financeiro no Home Care do Sistema Único de Saúde Métodos: Tratou-se de um estudo descritivo do tipo estudo de caso, que se utilizou da avaliação econômica em saúde. Os dados foram coletados em uma matriz e compilados no programa Excel onde foram analisados. Foi submetido e aprovado no Comitê de ética em Pesquisa. Resultados: Constatou-se um percentual elevado de contas médicas glosadas para as doenças crônicas não transmissíveis que vêm ocupando o topo da lista dos custos em saúde. As glosas evidenciadas neste estudo demonstraram o cenário dos custos no homecare específico no SUS, representando os itens e subitens mais glosados, as suas justificativas e a apresentação financeira do período estudado. Conclusão: Com base neste estudo, constatou-se um percentual considerado elevado de contas médicas para as doenças crônicas não transmissíveis. A auditoria em saúde vem buscar, contribuir e melhorar o equacionamento da gestão otimizando uma melhor a alocação de recursos financeiros em saúde.

Palavras-chave: Auditoria financeira, Custos, Pagamento, Assistência domiciliar.

\begin{abstract}
Objective: To analyze the disallowances and their justifications as their financial impact on the Home Care of the Unified Health System Methods: It was a descriptive study of the case study type, which used the economic evaluation in health. The data were collected in a matrix and compiled in the Excel program where they were analyzed. It was submitted to and approved by the Research Ethics Committee. Results: There was a high percentage of disallowed medical bills for chronic non-communicable diseases that have been at the top of the list of health costs. The disallowed evidenced in this study demonstrated the scenario of costs in the specific homecare in SUS, representing the most disallowed items and subitems, their justifications and the financial presentation of the period studied. Conclusion: Based on this study, a high percentage of medical bills for chronic non-communicable diseases was found. The health audit seeks, contributes and improves the management approach, optimizing the allocation of financial resources in health.
\end{abstract}

Keywords: Financial audit, Costs, Payment, Home care.

\section{RESUMEN}

Objetivo: Analizar las desestimaciones y sus justificaciones como su impacto financiero en la Atención domiciliaria del Sistema Único de Salud. Métodos: Este fue un estudio descriptivo del tipo de estudio de

1 Programa de Pós-Graduação em Enf. (UNIRIO), Rio de Janeiro - RJ. *E-mail: enfoadamcarlos@gmail.com 2 Escola de Enfermagem Alfredo Pinto (UNIRIO), Rio de Janeiro - RJ. 
caso, que utilizó la evaluación económica en salud. Los datos se recopilaron en una matriz y se compilaron en el programa Excel donde se analizaron. Fue presentado y aprobado por el Comité de Ética en Investigación. Resultados: Hubo un alto porcentaje de facturas médicas rechazadas por enfermedades crónicas no transmisibles que han estado en la parte superior de la lista de costos de salud. Las glosas evidenciadas en este estudio demostraron el escenario de costos en la atención domiciliaria específica en SUS, que representa los elementos y subpuntos más no permitidos, sus justificaciones y la presentación financiera del período estudiado. Conclusión: Según este estudio, se encontró un alto porcentaje de facturas médicas por enfermedades crónicas no transmisibles. La auditoría de salud busca, contribuye y mejora el enfoque de gestión, optimizando una mejor asignación de recursos financieros en salud.

Palabras clave: Auditoría financiera, Costos, Pago, Cuidados en el hogar.

\section{INTRODUÇÃO}

Home health care ou também conhecido como home care, é a atenção à saúde na assistência domiciliar, atendimento hospitalar domiciliar e a internação domiciliar, são diferentes tipos de denominações de uma forma de atendimento em saúde de saúde cada vez mais frequente nos dias de hoje, onde se presta atendimento na casa, domicílio ou o lar do paciente (SAMPAIO AVCF e IMAI C, 2012).

Sampaio AVCF e Imai C (2012) descrevem que o home care é utilizado para atendimento ambulatorial como para internação domiciliar, sendo que no caso de internamento, direciona ao tratamento multidisciplinar especializado com atendimento 24 horas por uma equipe médica-enfermagem no domicílio do paciente. Contextualizando a problemática do estudo, o Ministério da Saúde (BRASIL, 2016) define, no âmbito do SUS, a internação domiciliar como um conjunto de atividades prestadas no domicílio a pessoas clinicamente estáveis que exijam intensidade de cuidados acima das modalidades ambulatoriais, mas que possam ser mantidas em casa, por equipe exclusiva para esse fim.

Busmester H e Morais MV (2014) colocam que a desospitalização precoce é uma tendência mundial, não só para minimização de custos, mas também porque já está demonstrado que, para determinadas propostas de tratamento, a recuperação da doença é mais rápida e efetiva em ambiente familiar.

A auditoria é um processo sistemático, documentado e independente de se avaliar objetivamente uma situação ou condição para determinar a extensão na qual critérios são atendidos, obter as evidências quanto a esse atendimento e relatar os resultados dessa avaliação a um destinatário predeterminado (BOLETIM DO TRIBUNAL DE CONTAS DA UNIÃO, 2011a). No entendimento do Ministério da Saúde (BRASIL, 2013) a auditoria é um instrumento de gestão para fortalecer o Sistema Único de Saúde (SUS), contribuindo para a alocação e utilização adequada dos recursos, a garantia do acesso e a qualidade da atenção à saúde oferecida aos cidadãos.

O Departamento Nacional de Auditoria do SUS (DENASUS) (2004) coloca que algumas inconformidades são identificadas no processo de avaliação das contas em saúde, que podem ocasionar a recusa de pagamento parcial ou total. Na ocorrência de glosas, recomenda-se verificar toda a documentação clínica, para que as inconformidades sejam apresentadas e fundamentadas, de acordo com as legislações vigentes e os protocolos assistenciais da época desta análise (SILVA ACC, 2018). .

Rodrigues JARM (2018) descreve que a auditoria de contas hospitalares é uma ferramenta controladora de custos, sendo utilizado pelos hospitais para o monitoramento da sua área financeira. Ressalta-se a importância do controle destes processos pelos fluxos de documentos utilizados, e que a equipe de enfermagem e de saúde, devem estar ambientada para interagirem com estes processos, facilitando a auditoria externa que será realizada posteriormente.

O DENASUS (2005) conceitua a glosa como a rejeição total ou parcial de recursos financeiros cobrados pela parte contratada, porém a aplicação é realizada pela contratante com motivos baseados em documentação clínica adequada, comprovando com base técnica o ato aplicado. 
O DENASUS (2004) direciona que durante a análise da auditoria da prestação dos serviços assistenciais ao SUS, a glosa total, somente deverá ser aplicada após toda a verificação dos documentos que dão suporte ao processo de auditagem, como os livros de ocorrência dos setores por onde o paciente esteve internado, livros de admissão e alta da unidade, check-list de gastos de centro-cirúrgico ou do setor de internação e até uma verificação direta com o paciente e seus familiares como uma simples entrevista ou o envio de carta registrada caso a alta já tenha ocorrido.

Saber os índices de glosas e os seus custos é considerado importante, pois permite uma avaliação técnica do processo de faturamento apresentado pela empresa para o pagamento dos serviços prestados durante a internação domiciliar, confirmando o trabalho realizado pela equipe de auditoria durante a análise perante a alocação de recursos financeiros em saúde (SILVA ACC, 2018).

Entender esse processo é importante, pois orientam os gestores federais, estaduais e municipais nas informações suficientes, instrumentos de regulação e avaliação para realizar uma contratação adequada às necessidades de serviços de saúde de sua população (BRASIL, 2001).

As glosas surgem normalmente após algumas falhas durante o processo de atendimento operacional, assistencial e do próprio faturamento das empresas que prestam o serviço, devendo o órgão contratante estar atento a essas possíveis falhas durante o processo de avaliação das contas saúde dos serviços prestados pelas empresas terceirizadas (SILVA ACC, 2018).

Diante de qualquer glosa realizada pelo contratante, qualquer empresa contratada pode solicitar as justificativas da glosa, seja ela técnica ou administrativa e, por meio da solicitação de recurso, oficialmente contesta ou acata os itens glosados junto aos auditores. Essa revisão nos valores glosados é permitida por lei, e deverá incluir todas as justificativas das glosas, prazos para a sua contestação e o tempo em que será realizado o retorno das solicitações (SILVA ACC, 2018).

A Agência Nacional de Saúde Suplementar (ANS) (2014), é uma autarquia sob regime especialque está vinculada ao Ministério da Saúde, coloca que não se pode estabelecer nenhuma negativa de contestação de glosa via contratual, pois todo o prestador deverá conhecer os motivos que levaram a recusa de pagamento dos itens e subitens cobrados em conta.

Reforçam-se ainda, que todo o contrato realizado entre as partes, deverá conter os casos de glosa, prazos para a entrada de recursos e o tempo de resposta da operadora (AGÊNCIA NACIONAL DE SAÚDE SUPLEMENTAR, 2014).

As inconformidades identificadas que geraram as glosas, total ou parcial, ocorrem quando em relação às regras e práticas adotadas pelo Home Care, divergem das preconizadas pelas legislações vigentes. Isso representa um embate na relação contratual estabelecida entre as partes, sendo necessário um recurso de glosa para equalizar a relação contratual com retorno ou não das ações aplicadas (SILVA ACC, 2018).

O indicador da glosa, ou a recusa do pagamento, apóia o gestor, no sentido de detectar erros que irão sobrecarregar os custos e impedir a promoção de um serviço de qualidade, pois a auditoria favorece que se atinjam metas dentro dos sistemas de saúde (ROSA MR, 2017).

O aumento crescente nos custos em saúde vem trazendo preocupação aos profissionais e, sobretudo, aos gestores que atuam nessa área no que diz respeito à adoção de estratégias para controle de gastos, a equipe de Auditoria no SUS diante de um cenário de transição epidemiológica e da escassez de recursos públicos visa garantir a eficiência alocativa dos recursos (SILVA ACC, 2018).

Francisco IMF e Castilho V (2012) afirmam que custos crescentes e recursos escassos têm afetado todos os prestadores de serviços de saúde, e que não houve outro momento em que as organizações de saúde tiveram de operar com mais eficiência e ter mais cuidado com as restrições de custos como na atualidade.

Custo representa tudo o que é gasto direta ou indiretamente na produção de um bem ou na prestação de um serviço. Os custos podem ser classificados em diretos, indiretos ou de transformação. Os primeiros representam os gastos diretos na produção de um serviço, representados por materiais e mão de obra 
direta. Os custos indiretos são comuns a diversos serviços, necessitando de rateio entre os setores envolvidos para a atribuição de valores. Já os de transformação referem-se àqueles dispensados na aquisição de um produto (CASTILHO V e JERICÓ MC, 2010; PEREIRA FV e SCHUTZ V, 2012).

Dando prosseguimento, os autores enfatizam que as instituições públicas ou privadas que atuam na produção de serviços de saúde utilizam inúmeros insumos para execução de suas atividades, incluindo recursos materiais e humanos, que são conhecidos como itens de custo.

Os itens de custo representam todos os gastos decorrentes da produção de serviços. O custo com estes itens é pago diretamente pelo cliente, indiretamente através das operadoras de saúde ou, principalmente, pelo financiamento do setor público (BRASIL, 2013; CASTILHO V e JERICÓ MC, 2010). Diante dos estudos mencionados, esse estudo obteve como objetivo analisar as glosas e as suas justificativas no Home Care do Sistema Único de Saúde.

\section{MÉTODOS}

Tratou-se de um estudo descritivo, do tipo estudo de caso, que se utilizou da avaliação econômica em saúde para analisar as glosas e as suas justificativas como o seu impacto financeiro no Home Care do Sistema Único de Saúde no município norte fluminense.

A escolha da instituição ocorreu devido ao órgão demandar estudos sobre glosas e custos durante a prestação de serviços na internação domiciliar. O Serviço de Auditoria do Município funciona como parte do Departamento de Auditoria, Controle e Avaliação da Secretaria Municipal de Saúde. Neste estudo, as modalidades do Serviço de Internação Domiciliar (SID) foram divididas em três grupos, um (paciente de baixa complexidade), dois (média complexidade) e três (alta complexidade).

A amostra do estudo foi composta pelas contas médicas dos pacientes internados no SID durante a prestação do serviço de janeiro a outubro do ano de 2015. Foram consideradas todas as contas, independente de tempo de permanência de internação, que contabilizou um total de 605 faturas. $O$ estudo foi submetido e aprovado pelo Comitê de Ética em Pesquisa (CEP) da Universidade Federal do Estado do Rio de Janeiro (UNIRIO), enquanto instituição proponente, que serviu de base para o município norte fluminense tendo recebido o número de registro CAAE 62231416.3.00005285 e o número de Parecer 1.949.693.

Os dados foram compilados no programa excel e os resultados apresentados como gráfico e tabelas. A análise dos objetivos específicos do estudo foi realizada por meio da estatística descritiva, que é utilizada para descrever e resumir os dados e, desta forma, tirar conclusões sobre eles.

\section{RESULTADOS E DISCUSSÃO}

Foram analisadas as glosas baseadas nas doenças e na modalidade do SID de acordo com a frequência e o custo. Dentre as glosas mais evidenciadas no estudo estão às sequelas de doenças cerebrovasculares (35\%), seguidas por outros transtornos do encéfalo $(14,8 \%)$, traumatismo dos nervos cranianos $(9,1 \%)$ e Doença de Alzheimer (8,46\%) (SILVA ACC, 2018).

As doenças mais frequentes envolvidas nessas modalidades de gerenciamento são: diabetes, hipertensão arterial, doenças respiratórias crônicas, doenças vasculares, tanto cardíacas como as neurológicas, as nefropatias crônicas e doenças osteomusculares (BUSMESTER H e MORAIS MV, 2014).

De acordo com Silva ACC (2018) os tipos da modalidade do SID no município, observa-se a 2 com $62,6 \%$ com a maior frequência de glosas dentre todas as pesquisadas, seguidas pela a $1 \mathrm{com} 32,4 \%$ e a 3 com $5 \%$. Ao verificar o custo das glosas por modalidade, a 2 representa a maior delas, porém com maior distribuição de pacientes e de contas médicas neste tipo (69,74\%), seguidas pela $1 \mathrm{com} 22,37 \%$ e a pela 3 com $7,89 \%$ dos pacientes atendidos.

O envelhecimento populacional e, consequentemente, o tratamento das DCNT, são fatores que geram maior utilização do sistema de saúde. Entre as principais e primeiras intervenções, é necessário conhecer 
os componentes da carteira de serviços em saúde, identificar sua condição de saúde e determinar planos de cuidados que reduzam os agravos e as suas complicações (BUSMESTER H e MORAIS MV, 2014). Conforme na Tabela 1, a variável sobre o custo das glosas no SID, analisou o percentual glosado de acordo com o tipo de modalidade:

Tabela 1 - Custos das glosas por modalidade no SID.

\begin{tabular}{cc}
\hline Variáveis & Valores \\
\hline Modalidade 1 & $\mathrm{R} \$ 192.436,51$ \\
Modalidade 2 & $\mathrm{R} \$ 238.786,81$ \\
Modalidade 3 & $\mathrm{R} \$ 22.673,78$ \\
\hline Total geral & $\mathrm{R} \$ 453.897,10$ \\
\hline Fonte: Silva ACC e Schutz V, 2018.
\end{tabular}

O maior custo no SID está inserido na modalidade $2(\mathrm{R} \$ 238.786,81)$, porém a maioria dos pacientes inseridos neste tipo com $62,6 \%$ representando o maior número de contas médicas para a média complexidade, seguida pela modalidade $1(\mathrm{R} \$ 192.436,51) \mathrm{com} 32,4 \%$ de pacientes de baixa complexidade e a modalidade $3(R \$ 22.673,78)$ com $5,0 \%$ de paciente de alta complexidade (SILVA ACC, 2018). Para a análise das contas médicas e hospitalares, a cobrança é mais consistente quando feita separadamente.

Para cada componente do custo, cada divergência observada deve ser comunicada ao prestador, na forma de relatório formal e justificado, a fim de que este possa, por meio de recursos, esclarecer ou justificar o que estiver sendo cobrado (BUSMESTER H e MORAIS MV, 2014).

Silva ACC (2018) coloca a importância das ações da auditoria nos locais de internação domiciliar, focando as visitas aos pacientes em seus domicílios, analisando o uso de equipamentos, os materiais e os medicamentos utilizados, as evoluções multidisciplinares etc.

As glosas e os seus custos foram analisados de acordo com a variável dos itens de custo, representando o percentual que mais glosados e menos glosados conforme o seu uso, demonstrando o perfil desta análise conforme a Tabela 2.

Tabela 2 - Frequência das glosas dos itens de custo.

\begin{tabular}{cc}
\hline Item glosado & Frequência \\
\hline Apoio Respiratório & $21,30 \%$ \\
Atendimento Profissional & $7,96 \%$ \\
Dieta & $1,17 \%$ \\
Exames & $0,13 \%$ \\
Indeterminado & $0,11 \%$ \\
Material & $38,27 \%$ \\
Medicamento & $25,01 \%$ \\
Mobiliário & $4,42 \%$ \\
Sem glosa & $1,63 \%$ \\
\hline Total Geral & $100 \%$ \\
\hline
\end{tabular}

Fonte: Silva ACC e Schutz V, 2018.

Oliveira AD, et al (2014) apresentam em seu estudo que os materiais e medicamentos são os itens de contas hospitalares que mais impactam o total de glosas numa instituição. Conforme as glosas efetuadas pelos auditores, os materiais e medicamentos são os que mais ocorrem na instituição.

Ao comparar com os achados desta pesquisa, observam-se os mesmos resultados, em que os materiais e medicamentos sãos os itens em conta mais glosados pelos auditores. Somando ambos os itens, 
representam mais de $50 \%$ das glosas realizadas. Reforça-se a análise e a pertinência do uso de materiais e medicamentos em todos os pacientes do SID (SILVA ACC, 2018). Na Tabela 3, que versa sobre a frequência das glosas de acordo com os subitens em conta médica, descreveu os mais glosados, que representou $47,9 \%$ conforme descrito:

Tabela 3 - Frequência das glosas dos subitens em conta.

\begin{tabular}{cc}
\hline Subitem Glosado & Frequência (\%) \\
\hline Compressa não Estéril 7,5 x 7,5 & $16,8 \%$ \\
Oxigênio e Aspiração & $8,6 \%$ \\
Aspirador Elétrico Portátil & $4,9 \%$ \\
Sonda de Aspiração Traqueal & $4,3 \%$ \\
Acetilcisteína 600mg Envelope & $3,8 \%$ \\
Nebulizador & $3,5 \%$ \\
Supervisão de Enfermagem & $3,1 \%$ \\
Seringa Descartável 20 ml & $2,9 \%$ \\
\hline Total Geral & $47,9 \%$ \\
\hline
\end{tabular}

Fonte: Silva ACC e Schutz V, 2018.

Os demais subitens glosados que não foram apresentados nesta tabela, quando somados resultaram em $52,1 \%$ do percentual de glosas, e pelo grande número apresentados não foram descritos, porém com menores percentuais dos colocados acima (SILVA ACC, 2018).

Ao identificar a compressa não estéril $7,5 \times 7,5 \mathrm{~cm}$ no topo da Tabela 3 , com $16,8 \%$ do percentual glosado na maioria dos meses pela equipe de auditores do município, pode-se questionar o motivo pelo qual este material é o recordista da frequência de glosa (SILVA ACC, 2018).

É importante lembrar que, em uma análise de representatividade de custos, por exemplo, nem sempre o procedimento ou o caso que aparece no topo da lista é o de maior custo, pois a combinação dos dois fatores - frequência e custo - pode colocar um procedimento de baixo custo como gerador de custos elevados em uma determinada carteira, por ter frequência elevada. Daí a relevância de a auditoria analítica ter conhecimento do processo de atenção para validar a informação e propor ações que minimizem custos sem prejudicar a qualidade do atendimento (BUSMESTER H e MORAIS MV, 2014).

Oliveira AD, et al. (2014), em seu estudo sobre as glosas de materiais e medicamentos em um hospital privado na cidade de Brasília, demonstraram que o componente material recebeu o maior número de glosas (65\%), seguido também pelo item medicamento com $35 \%$ de glosas. Essa pesquisa corroborou com os achados nesta pesquisa, reforçando a importância da glosa como um indicador financeiro.

Dessa forma, no componente material, os subitens que mais tiveram representatividade foram os equipamentos de proteção individual (50\%), esparadrapo, compressa e álcool (10\%). No item medicamento, os componentes que receberam maior número de glosas foi o antibiótico (80\%), seguido pela Dipirona (12\%) e o medicamento Omeprazol com 4\% (OLIVEIRA AD, et al., 2012).

Observa-se no estudo mencionado a relação direta com os achados neste estudo, tendo o item material e a compressa gaze não estéril como o subitem de maior frequência nas glosas evidenciadas, porém de menor custo (SILVA ACC, 2018).

Ao comparar com o item medicamento, não se observou nenhuma relação direta com este estudo, pois se identificou como os mais glosados os subitens, a acetilcisteína envelope $600 \mathrm{mg}$ (3,8\%), Domperidona suspensão $100 \mathrm{ml}$ (2,8\%) e o Brometo de Ipratrópio frasco de $20 \mathrm{ml}$ (2\%) (SILVA ACC, 2018).

De acordo com as glosas observadas nos itens e subitens de custo, a Tabela 4, informa as justificativas de cada tipo existente encontrados nas análises da equipe de auditoria em saúde: 
Tabela 4 - Frequência das justificativas das glosas.

\begin{tabular}{cc}
\hline Justificativa da Glosa & Frequência \\
\hline Cobrança em excesso & $38,8 \%$ \\
Cobrança Indevida & $21,6 \%$ \\
Diferença de Valor & $16,2 \%$ \\
Não evoluído & $9,2 \%$ \\
Não checado & $7,1 \%$ \\
Não prescrito & $2,0 \%$ \\
Não consta em contrato & $2,0 \%$ \\
Sem glosa & $1,6 \%$ \\
Diferença de valor/Cobrança em excesso & $0,3 \%$ \\
Medicamento suspenso & $0,2 \%$ \\
Horário rodelado & $0,2 \%$ \\
Cobrança de preço de caixa & $0,2 \%$ \\
Apenas em SOS & $0,2 \%$ \\
Diferença de Valor/Não checado & $0,2 \%$ \\
Indeterminado & $0,1 \%$ \\
Duplicidade de cobrança & $0,1 \%$ \\
Total Geral & $100,0 \%$ \\
\hline
\end{tabular}

Fonte: Silva ACC e Schutz V, 2018.

Oliveira AD, et al. (2014), as falhas, em sua grande maioria, são causadas por erros de checagem da equipe de enfermagem, não precisamente pela não utilização do medicamento pelo paciente. Ascari RA (2011) no estudo de auditoria em enfermagem mostrou que, entre as inconsistências encontradas nos registros de enfermagem, estão a ausência de folhas de gastos da realização de procedimentos, ausência do tipo de cateter para a oxigenoterapia, uso excessivo de materiais, medicação não checada, uso de material não justificado, realização de exames sem pedido médico e administração de medicamento genérico sem o devido registro no prontuário quando prescrito pelo outro pelo médico assistente, o que gerou uma diferença em reais, de $\mathrm{R} \$ 761,27$ de glosa apresentada nesta fatura específica.

Estudos de Rosa MR (2017) descrevem que em 200 prontuários avaliados, 467 glosas foram encontradas, sendo a maioria relacionada à prescrição médica e registros de enfermagem. Dos itens mais glosados como a média de custos, foi possível identificar os procedimentos remunerados, sendo, sobretudo o oxigênio e as nutrições especiais.

O não cumprimento de procedimentos de checagem e da própria evolução destes procedimentos torna a glosa um componente frequente. As 31 glosas mais encontradas das contas auditadas em relação aos custos dos procedimentos remunerados são o curativo, o Haemoglucotest, nebulização, sondagens, oxigênios, equipo (ROSA MR, 2017).

Dantas IC, et al. (2017) descreveu que as glosas representam importante problema na auditoria em saúde e de enfermagem em serviços de Home Care. Os resultados da revisão de literatura apontam como principais causas de glosas a falta de checagem na prescrição médica e de enfermagem, desatenção na observância à evolução descrita nos registros dos pacientes, dificuldade na interpretação devido à letra ilegível e rasuras, falta de registro de procedimento de fisioterapia, falta de registros dos antibióticos, incompletude nos horários e datas nas evoluções e ausência da assinatura, número do registro e carimbos nas evoluções.

Em comparação ao estudo realizado, pode-se observar a relação da cobrança em excesso, a cobrança indevida, o não evoluído, o não checado, dentre outras justificativas que dão base para as inúmeras falhas identificadas dentre as justificativas dos itens mais glosados em relação ao custo.

Com ações voltadas para a educação continuada, treinamento e monitoramento das justificativas, esses indicadores encontrados podem ser minimizados, porém o envolvimento da equipe e dos gestores é importante para a melhoria dos resultados. 
Seja qual for o sistema de saúde no mundo, seja ele público ou privado, a implantação da equipe de auditoria em saúde é essencial para esse controle, nas suas várias vertentes apresentadas neste trabalho, que apresenta uma importante ferramenta na gestão da saúde, uma vez que nem sempre os gestores dispuseram das informações necessárias para decidir com segurança e consistência (GUEDES DN, et al., 2012).

$\mathrm{Na}$ relação apresentada de quantidade de faturas total versus faturas glosadas, pode-se observar na evolução retrospectiva de janeiro a outubro de 2015 , um percentual elevado de glosas nas faturas (Gráfico 1). Diversos meses ultrapassam o percentual de $85 \%$ de glosas, e alguns deles, como setembro e outubro, em especial, chegam próximo de $100 \%$ das faturas apresentadas.

Gráfico 1 - Apresentação de fatura x Faturas glosadas.

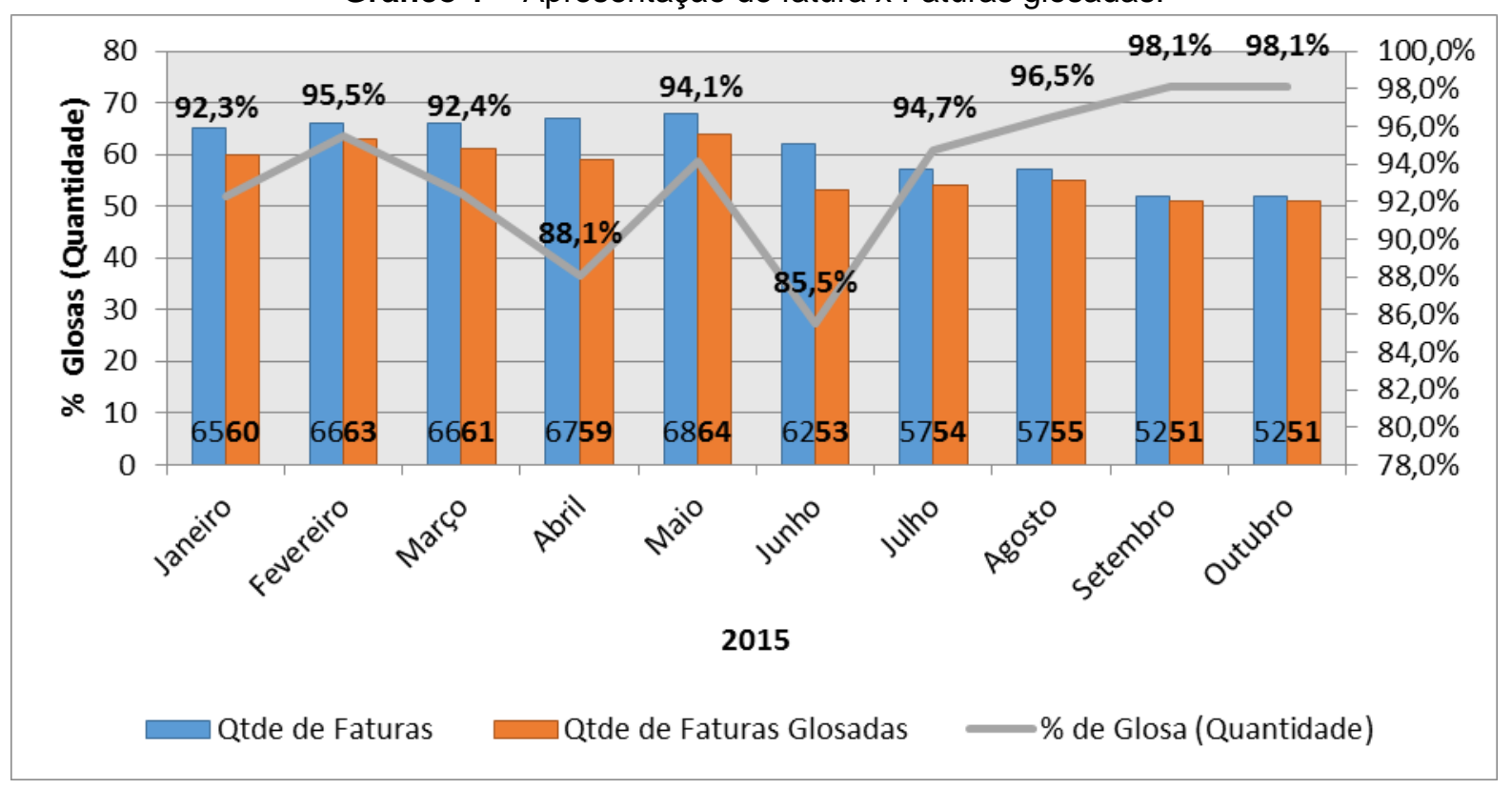

Fonte: Silva ACC e Schutz V, 2018.

Estudos de Rosa CDP e Santos MP (2013) relatam que na distribuição das contas recebidas e glosadas pela operadora ao hospital particular, nos meses de abril e maio de 2012, no primeiro mês houve uma apresentação total de $(R \$ 1.010 .485,70)$ que após análise da auditoria representou uma frequência total de glosas de $88,94 \%$ em todas as contas $(R \$ 125.658,91)$. No mês seguinte, uma apresentação ( $R \$$ $955.489,49)$ com $93,79 \%$ de glosas em todas as contas $(R \$ 63.251,94)$.

Observa-se a atuação frequente da auditoria também neste processo das auditorias de contas médicas, que direcionam as glosas mais evidentes, a média de custos destas glosas, os tipos de itens e subitens glosados e as suas justificativas.

Isso reforça com ênfase as adequações dos procedimentos às cobranças adequadas, sem deixar de registrar qualquer inconformidade existente nas contas apresentadas ao pagador. Isso apenas ilustra a grande eficiência que a auditoria pode representar a qualquer sistema, direcionado de forma adequada os recursos financeiros disponíveis (SILVA ACC, 2018).

Segundo Mendonça BCC (2005) para o SUS, a eficiência é estabelecida na relação entre os produtos (bens e serviços) gerados por uma atividade e os seus custos dos insumos empregados em um determinado período de tempo. O resultado que obtiver, vai expressar o custo de uma unidade de produto final em um determinado período de tempo.

Com esses itens glosados conforme citado neste estudo, observa-se um impacto orçamentário positivo para a operadora em questão, o que pode representar um balanço financeiro adequado para o investimento em outras ações em saúde. 


\section{CONCLUSÃO}

Com base neste estudo, constatou-se um percentual considerado elevado de contas médicas para as doenças crônicas não transmissíveis (DCNT), que vêm ocupando sistematicamente o topo da lista dos custos em saúde, em função da utilização progressiva de materiais de alto custo e de novas tecnologias, de tratamentos progressivamente mais onerosos e de complicações inerentes a essas doenças, entre outros motivos que geram maior utilização do sistema de saúde. As glosas evidenciadas neste estudo demonstraram o cenário dos custos no Homecare específico no SUS, representando os itens e subitens mais glosados, as suas justificativas e a apresentação financeira do período estudado. Com a identificação destas inconformidades, ocorreu um retorno orçamentário positivo aos cofres públicos, que sendo bem avaliados puderam ser aplicados em outras áreas nos blocos de financiamento em saúde. Reforça-se que e a auditoria em saúde vem buscar, contribuir e melhorar o equacionamento da gestão otimizando uma melhor a alocação de recursos financeiros em saúde.

\section{REFERÊNCIAS}

1. ASCARI RA. Auditoria de enfermagem: o impacto financeiro dos registros de enfermagem em prontuário médicocirurgião. In: Seminário Internacional Sobre o Trabalho na Enfermagem: Biossegurança no trabalho da enfermagem perspectivas e avanços. 2011; 3 .

2. BRASIL, Ministério da Saúde. Portaria no 825, de 25 de abril de 2016. Redefine a Atenção Domiciliar no âmbito do Sistema Único de Saúde (SUS) e atualiza as equipes habilitadas. Diário Oficial da União, Brasília, DF, 26 abr. 2016.

3. BRASIL, Agência Nacional de Saúde Suplementar- ANS. Lei no 13.003, de 24 de junho de 2014. Dispõe sobre os planos e seguros privados de assistência à saúde, para tornar obrigatória a existência de contratos escritos entre as operadoras e seus prestadores de serviços. Diário Oficial da União, DF, 25 jun. 2014.

4. BRASIL, Ministério da Saúde. Portaria no 963, de 27 de maio de 2013. Redefine a Atenção Domiciliar no âmbito do Sistema Único de Saúde (SUS). Diário Oficial da União, Brasília, DF, 28 maio 2013.

5. BRASIL, Tribunal de Contas da União. Normas de Auditoria do Tribunal de Contas da União: revisão junho 2011. Ano 44, n. 12. Brasília, DF, 5 jul. 2011

6. BRASIL, Ministério da Saúde. Portaria n²04/GM, de 29 de janeiro de 2007. Regulamenta o 11- financiamento e a transferência dos recursos federais para as ações e os serviços de saúde, na forma de blocos de financiamento, com o respectivo monitoramento e controle. Diário Oficial da União, Brasília, DF, 30 jan. 2007.

7. BRASIL. Ministério da Saúde. Departamento Nacional de Auditoria do SUS. Orientações técnicas sobre aplicação de glosas em auditoria no SUS: caderno 1 / Ministério da Saúde, Departamento Nacional de Auditoria do SUS. Brasília: Ministério da Saúde, 2005. 88 p.: il. - (Série A. Normas e Manuais Técnicos) ISBN 85-334-0872-2

8. BRASIL, Ministério da Saúde. Secretaria Executiva. Departamento Nacional de Auditoria do SUS. Manual de Glosas do Sistema Nacional de Auditoria. Brasília, DF, 2004.

9. BRASIL, Ministério da Saúde. Secretaria de Assistência à Saúde. Orientações para a compra de serviços de saúde. Brasília, DF, 2001.

10. BUSMESTER H e MORAIS MV. Auditoria em saúde. São Paulo: Saraiva, 2014.

11. CASTILHO V e JERICÓ MC. Gerenciamento de custos: aplicação do método de custeio baseado em atividades em centro de material esterilizado. Rev. Esc. Enferm. USP. 2010; 44(3): 745-752, 20.

12. DANTAS IC, et al. Registro de enfermagem em home care: subsídios da auditoria de enfermagem. Rev. Enfermagem Contemporânea, Salvador, v. 6, n. 2, 2017.

13. FRANCISCO IMF e CASTILHO V. A enfermagem e o gerenciamento de custos. Rev Esc Enferm USP. 2002; 36(3): 240-4.

14. OLIVEIRA AD, et al. Glosas de materiais e medicamentos em um hospital privado na cidade de Brasília - Distrito Federal. Acta de Ciências e Saúde, [s. I.], 2012; 2(1).

15. PEREIRA FV e SCHUTZ V. Análise parcial de custos de materiais hospitalares: o custo de materiais hospitalares utilizados em clientes com insuficiência cardíaca dentro de uma enfermaria cardiovascular. R. Pesquisa: Cuid. fundam. (Online), Rio de Janeiro. 2012; 4(2): 2973-80.

16. RODRIGUES JARM, et al. Glosas em contas hospitalares: um desafio à gestão. Rev. Bras. Enferm. 2018; 71(5).

17. ROSA MR. Padronização da gestão do processo de auditoria interna em um hospital privado na cidade de Santa Catarina. 2017. 55 f. Dissertação (Mestrado em Enfermagem) - Programa de Pós-Graduação em Enfermagem, Universidade do Vale do Rio dos Sinos, São Leopoldo, 2017.

18. ROSA CDP e SANTOS MP. Auditoria de contas hospitalares: análise dos principais motivos de glosas em uma instituição privada. São Paulo. Anais. São Paulo: Administração da Produção, Logística e Operações Internacionais, 2013.

19. SAMPAIO AVCF e IMAI C. Atenção domiciliar à saúde (Home Health Care), a arquitetura acessível e o desenho universal. Nono Seminário Internacional Núcleo de Pesquisa em Tecnologia de Arquitetura e Urbanismo da Universidade de São Paulo, BRICS e a habitação coletiva sustentável, São Paulo, 2012.

20. SILVA ACC. Auditoria como ferramenta de gestão para a eficiência alocativa de recursos financeiros no SUS: $O$ caso da Prefeitura de Campos dos Goytacazes. -- Rio de Janeiro, 2018. 